Itinéraires Itinéraires

Littérature, textes, cultures

2015-2 | 2016

Stumbling blocks. Entraves et obstacles aux circulations

\title{
Situations de blocages dans la sphère de la protection de l'enfance : le statut particulier des enfants amérindiens aux États-Unis
}

Obstacles in Child Welfare: the Special Status of Native American Children in the United States

\section{Céline Planchou}

\section{(2) OpenEdition}

Journals

Édition électronique

URL : http://journals.openedition.org/itineraires/2911

DOI : 10.4000/itineraires. 2911

ISSN : 2427-920X

Éditeur

Pléiade

Référence électronique

Céline Planchou, «Situations de blocages dans la sphère de la protection de l'enfance : le statut particulier des enfants amérindiens aux États-Unis », Itinéraires [En ligne], 2015-2 | 2016, mis en ligne le 15 février 2016, consulté le 02 mai 2019. URL : http://journals.openedition.org/itineraires/2911 ; DOI : 10.4000/itineraires.2911

Ce document a été généré automatiquement le 2 mai 2019.

\section{cc) (†)}

Itinéraires est mis à disposition selon les termes de la licence Creative Commons Attribution - Pas d'Utilisation Commerciale - Pas de Modification 4.0 International. 


\title{
Situations de blocages dans la sphère de la protection de
} l'enfance : le statut particulier des enfants amérindiens aux États-Unis

\author{
Obstacles in Child Welfare: the Special Status of Native American Children in the \\ United States
}

Céline Planchou

1 Le statut amérindien aux États-Unis confère aux individus identifiés comme tels, et aux territoires sur lesquels ils résident, une dimension singulière au sein de la nation américaine. Les réserves indiennes sont ainsi, encore aujourd'hui, des espaces à part. Elles constituent des enclaves à l'intérieur du territoire étasunien qui relèvent d'un ordre juridique particulier et sur lesquelles s'exerce l'autorité des institutions politiques des tribus. Les individus qui composent ces tribus ont eux aussi un statut à part. Ces derniers jouissent en effet d'une appartenance politique multiple puisqu'ils sont à la fois membres de la nation américaine et membres de "nations dans la nation» (Deloria et Lytle 1998). Ils relèvent à la fois du droit américain, mais également des normes de la tribu dont ils sont membres, et du droit fédéral s'appliquant exclusivement aux Amérindiens.

2 Traditionnellement, les attributs de la spécificité amérindienne sont conditionnés par la résidence dans la réserve. Autrement dit, lorsqu'un Amérindien demeure en dehors de la réserve, les barrières juridiques qui le distinguent du reste de la population tombent et il devient un citoyen américain comme les autres. Toutefois, les réserves ne sont pas des espaces homogènes. Ce sont plutôt des territoires qui se superposent au territoire des États fédérés. L'imbrication des espaces tribaux et des espaces fédérés, qui s'accompagne d'une imbrication des compétences des institutions tribales et des institutions fédérées, est particulièrement complexe dans la sphère de la protection de l'enfance. 


\section{Imbrication des dispositifs et des responsabilités}

3 En raison du statut multiple des enfants amérindiens aux États-Unis, qui sont citoyens américains, citoyens d'un État fédéré et membres tribaux, le devoir de protection à l'égard des mineurs amérindiens en danger repose à la fois sur les institutions américaines et sur les institutions amérindiennes, construisant un dispositif où les responsabilités sont pour le moins enchevêtrées. Tout d'abord, ces mineurs relèvent des mécanismes généraux de protection de l'enfance, à savoir ceux qui existent pour les enfants non indiens. Ces mécanismes émanent déjà de différents niveaux de gouvernement puisqu'ils reposent sur l'État fédéral et sur les États fédérés. Le premier détient une responsabilité principalement financière dont il s'acquitte grâce aux fonds disponibles par le biais du Social Security Act, en particulier du titre IV intitulé « Grants to States for Aid and Services to Needy Families with Children and for Child-Welfare Services ${ }^{1}$ ». C'est donc avant tout aux seconds, donc aux États, qu'incombe la responsabilité à l'égard des mineurs en danger au sein de la fédération américaine. La protection administrative de ces mineurs repose sur les services sociaux des États, qui sont en partie financés par les aides sociales fédérales, tandis que leur protection judiciaire est assurée par les tribunaux pour enfants.

4 Si les mineurs amérindiens relèvent bien de ces mécanismes généraux de protection de l'enfance, ils dépendent également de dispositifs spécifiques auxquels les enfants non indiens n'ont pas accès. Ces dispositifs impliquent les institutions tribales, en particulier les services sociaux et les tribunaux amérindiens, de même que le gouvernement fédéral. En effet, depuis la création des États-Unis, les affaires indiennes sont avant tout du ressort de l'État fédéral et sont placées sous la responsabilité du Bureau des affaires indiennes (BIA) au sein du ministère de l'Intérieur².

5 Selon le schéma juridictionnel traditionnel, organisé autour de la résidence, un enfant amérindien habitant dans une réserve devrait relever du dispositif de protection de l'enfance spécifique qui implique les institutions tribales et l'administration fédérale en charge des affaires indiennes, c'est-à-dire le BIA. Les enfants qui demeurent en dehors des réserves devraient, pour leur part, être du ressort du dispositif général de protection de l'enfance qui repose en premier lieu sur les États. Toutefois, selon les époques, l'un ou l'autre des dispositifs a été privilégié par l'État fédéral, redessinant ainsi constamment les relations de pouvoir entre les différents acteurs gouvernementaux impliqués et faisant évoluer les contours des barrières juridiques qui existent entre les réserves et le reste du territoire étasunien et entre les enfants amérindiens et les citoyens américains non indiens.

\section{Les barrières juridiques tombent et les placements augmentent}

6 Au milieu du $\mathrm{xx}^{\mathrm{e}}$ siècle, l'administration fédérale en charge des affaires indiennes décide de se désengager de la sphère de la protection de l'enfance. Elle développe ainsi une politique en la matière selon laquelle l'intérêt des enfants amérindiens serait d'être traité comme tous les autres mineurs américains. Insistant sur le fait que ces enfants sont avant tout des citoyens américains, le BIA souhaite alors transférer la responsabilité à leur 
égard du dispositif spécifique vers le dispositif général de protection de l'enfance (Pryse 1951). Cette politique se solde par une implication grandissante des services de protection de l'enfance des États dans les familles amérindiennes, où que ces dernières résident sur le territoire national, tandis que les gouvernements tribaux sont de plus en plus écartés des décisions affectant leurs membres mineurs les plus vulnérables (Planchou 2012). Autrement dit, le découpage territorial des États prévaut sur celui des réserves alors que l'organisation administrative et judiciaire des États vient progressivement ordonner l'action sociale en faveur des enfants amérindiens, même lorsque ces derniers résident dans une réserve. Les barrières juridiques construites par le statut particulier des territoires et des individus amérindiens tombent alors progressivement. Elles ne représentent plus des obstacles à la circulation des enfants, lesquels sont placés de plus en plus souvent en dehors des réserves par les services sociaux des États dans des familles d'accueil ou d'adoption non indiennes.

7 Pourtant, ce glissement des enfants amérindiens d'un dispositif de protection de l'enfance à un autre n'a jamais été sanctionné par une loi du Congrès. Il n'a jamais été juridiquement entériné, mais s'est produit de fait, au gré de programmes et de pratiques qui sont alors mis en œuvre au sein de l'administration fédérale en charge des affaires indiennes et des États. L'Indian Adoption Project est ainsi lancé conjointement par le BIA et la Child Welfare League of America en 1958 (Fanshel 1972, Brieland 1973, Palmiste 2011, Planchou 2012). Ce programme est en fait un projet pilote destiné spécifiquement aux enfants indiens et conçu pour expérimenter deux types d'adoption qui, pense-t-on alors, doivent pouvoir bénéficier à tous les enfants américains en danger. C'est d'une part l'adoption «interraciale » (transracial) ${ }^{3}$ et, d'autre part, l'adoption "interfédérée » ( interstate $)^{4}$, c'est-à-dire d'un État à un autre. Les enfants qui participent au projet, lesquels résident pour la plupart dans une réserve, doivent ainsi non seulement être adoptés par des couples non indiens, mais également au sein d'un État différent de celui dont ils sont originaires. Ils sont donc exclusivement identifiés en fonction de l'État d'où ils viennent et non en fonction de leur affiliation tribale. Leur statut spécifique ainsi mis de côté, il est alors plus aisé de faire intervenir les institutions des États, en particulier les tribunaux pour enfants et, dans le même temps, d'écarter les gouvernements tribaux. En 1967, lorsque le projet prend fin, 395 enfants ont été placés dans vingt-six États, surtout dans l'Est et le Middle West (Lyslo 1968). Par ailleurs, l'Indian Adoption Project a impulsé un mouvement plus large au sein de la société américaine en encourageant les agences d'adoption des États à étendre leurs services à des enfants qu'ils ne considéraient pas jusque-là comme relevant de leur responsabilité. Ainsi, indépendamment des enfants placés par le biais du projet, l'adoption des mineurs amérindiens augmente considérablement dans les années 1960 (Unger 1977).

Outre l'Indian Adoption Project et le mouvement qu'il a impulsé, certaines pratiques se développent alors qui participent également à favoriser la circulation des enfants amérindiens au-delà des frontières des réserves. Ainsi, il n'est pas rare au milieu du $\mathrm{xx}^{\mathrm{e}}$ siècle de tirer profit du statut de certaines terres individuelles pour contourner l'autorité des gouvernements tribaux. Comme nous l'avons précisé auparavant dans cet article, les réserves sont rarement des espaces homogènes. L'imbrication des espaces tribaux et des espaces fédérés remonte à la fin $\mathrm{du} \mathrm{XIX}^{\mathrm{e}}$ siècle, lorsque l'État fédéral met en place des politiques assimilationnistes qui cherchent à intégrer les Amérindiens à la nation américaine. Un aspect de ces politiques est d'imposer la propriété individuelle sur des espaces jusque-là détenus de manière collective. Suite au morcellement des réserves, 
lancé en 1887 par le Dawes Act ${ }^{5}$, les territoires indiens sont découpés en lopins qui, aux termes de recensements effectués parmi les différentes tribus, sont attribués à chaque individu. Tous ne bénéficient pourtant pas du même titre de propriété sur ce lopin de terre. Tandis que certains propriétaires sont jugés « incompétents » par l'administration fédérale en charge des affaires indiennes, et que leur lopin demeure sous la tutelle de l'État fédéral, d'autres sont, à l'inverse, jugés « compétents ${ }^{6}$ ». Leur terre perd alors son statut spécifique, c'est-à-dire qu'elle est toujours géographiquement située dans la réserve, mais qu'elle sort du régime foncier particulier, tombant ainsi sous la juridiction de l'État fédéré dans lequel elle se trouve ${ }^{7}$.

Ce sont ces terres, situées à la fois dans et en dehors des réserves, qui permettent, dans les années 1950, de contourner l'autorité des institutions tribales sur leurs membres mineurs. Ainsi, en 1960, le conseil tribal navajo adopte une résolution dénonçant l'action de certaines organisations non indiennes, notamment des groupes religieux, qui n'hésitent pas à s'installer sur ce type de terres afin de pouvoir recourir aux tribunaux d'État pour placer des enfants navajos loin de la réserve sans avoir à traiter avec la cour tribale navajo (conseil tribal navajo, 1960).

\section{L'Indian Child Welfare Act}

Le nombre d'enfants amérindiens retirés de leur famille et placés en familles d'accueil et d'adoption non indiennes ne cesse de s'accroître dans les années 1960. Au tournant des années 1970, des études menées par l'Association on American Indian Affairs démontrent que dans certains États à forte population amérindienne, 25 à $35 \%$ des mineurs autochtones ne vivent plus au sein de leur famille ${ }^{8}$. C'est alors que les parents amérindiens et les institutions tribales se mobilisent. Les premiers recourent de plus en plus aux tribunaux américains afin de contester des décisions de placement, tandis que les gouvernements tribaux affirment leur autorité sur leurs membres mineurs en adoptant des résolutions allant en ce sens, à l'instar de la résolution tribale navajo de 1960 , et en développant plus avant leurs dispositifs administratifs et judiciaires de protection de l'enfance.

11 La mobilisation amérindienne mène, en 1974, à la tenue d'une audience spéciale devant le sous-comité aux Affaires indiennes du Sénat américain dont le but est d'examiner «les problèmes auxquels les familles indiennes sont confrontées pour élever leurs enfants ainsi que la manière dont l'action ou l'inaction du gouvernement fédéral a eu un impact sur ces problèmes ${ }^{9}$ » (Subcommittee on Indian Affairs, 1975). De nombreux intervenants témoignent du fait que les décisions de placement émanant des services sociaux des États ne sont pas toujours justifiées et qu'elles reposent parfois sur des préjugés socioculturels. Ils estiment que les placements ne sont pas nécessairement la meilleure solution pour régler les problèmes socio-économiques qui pèsent sur les réserves mais qu'au contraire, ils contribuent à détruire davantage les familles indiennes au sein desquelles les solidarités ont été déjà largement érodées par des décennies de politiques fédérales assimilationnistes, en particulier la politique des pensionnats ${ }^{10}$. D'autre part, d'aucuns considèrent que ces placements violent le statut particulier des enfants amérindiens en ne reconnaissant pas le lien essentiel qui les relie à leur tribu.

12 Face à la mobilisation amérindienne, l'État fédéral ne reste pas sans réaction. L'arrêt Fisher v. District Court ${ }^{11}$ de 1976 puis, surtout, la loi fédérale sur les enfants amérindiens de $1978^{12}$ redonnent ainsi tout son sens au statut particulier des mineurs autochtones dans la 
sphère de la protection de l'enfance. La décision de la Cour suprême affirme tout d'abord le caractère exclusif de l'autorité des tribunaux amérindiens sur les procédures d'adoption ayant lieu dans une réserve. De son côté, l'Indian Child Welfare Act va plus loin puisqu'il s'applique aux adoptions, mais également aux placements en structures d'accueil, aux procédures de déchéance des droits parentaux et aux placements préadoptifs, c'est-à-dire les placements temporaires d'enfants en structures d'accueil après que les parents ont été déchus de leurs droits, mais avant une adoption plénière.

Tout en replaçant les institutions amérindiennes au centre des décisions affectant leurs membres mineurs, la loi fédérale de 1978 tente d'éclaircir la question de la répartition des compétences entre les institutions tribales et les institutions fédérées dans les quatre types de procédure cités précédemment. Un schéma juridictionnel est établi qui s'articule autour de la résidence de l'enfant. Si celui-ci réside dans une réserve, il relève de l'autorité exclusive de la tribu. S'il réside en dehors de la réserve, des compétences partagées sont dévolues aux cours tribales et aux instances judiciaires des États. Ainsi, lorsqu'une procédure évoquée ci-dessus impliquant un enfant amérindien est entamée au sein d'une institution d'État, cette institution doit tenir informée la tribu à laquelle l'enfant est affilié. Cette dernière a dès lors la possibilité d'obtenir un transfert de la procédure à la cour tribale. S'il n'y a pas transfert, la tribu peut tout de même continuer à intervenir en étant par exemple représentée lors des différentes audiences qui jalonnent la procédure au sein du tribunal d'État. En outre, si la procédure reste entre les mains des institutions de l'État, celles-ci sont désormais tenues de respecter un certain nombre de dispositions prévues par la loi. Lorsqu'il est prouvé qu'un placement est justifié et qu'il sert bien l'intérêt de l'enfant, les services sociaux fédérés doivent par exemple respecter un ordre de préférence afin de mener à bien ce placement. Dans le cas d'une adoption, l'Indian Child Welfare Act requiert qu'un enfant amérindien soit placé en premier lieu chez un membre de la famille élargie, puis chez un membre de la tribu, puis au sein d'une famille indienne et, en dernier recours, au sein d'une famille non indienne.

$14 \mathrm{Au}$ vu de ces éléments, il est évident que la possibilité pour des parents non indiens d'adopter des enfants amérindiens est désormais fortement limitée. En plus du consentement écrit des parents biologiques dans le cas d'une adoption dite volontaire, c'est-à-dire lorsque l'adoption ne fait pas suite à une procédure de déchéance des droits parentaux mais qu'elle intervient lorsqu'un parent a fait le choix de renoncer à ses droits pour que l'enfant soit adopté, la tribu doit être tenue au courant et peut s'y opposer. Des barrières juridiques entre les enfants amérindiens et les parents adoptifs non indiens existent bien aujourd'hui et sont difficilement surmontables. Elles sont la marque du statut particulier de ces enfants et de l'existence distincte des tribus auxquelles ils appartiennent.

\section{Des blocages pour accéder aux aides sociales fédérales}

15 En plus de redonner son sens au statut particulier des enfants amérindiens en affirmant l'autorité des gouvernements tribaux sur leurs membres mineurs en danger, la loi fédérale de 1978 entend appuyer ces gouvernements dans la mise en œuvre de leurs propres programmes de protection de l'enfance. Pour ce faire, des fonds spécifiques sont débloqués, sous la forme de subventions allouées par le BIA. Ces fonds sont toutefois limités et sont fortement réduits dès le début des années 1980. Les gouvernements 
tribaux n'ont par ailleurs pas accès aux aides sociales fédérales disponibles par le biais du Social Security Act. C'est en effet le BIA, et non l'administration fédérale en charge des affaires sociales, qui détient le pouvoir de traiter directement avec les tribus au sein de l'appareil administratif fédéral ${ }^{13}$. Autrement dit, la circulation des aides sociales fédérales destinées aux enfants en danger est entravée puisque ces aides ne parviennent pas jusqu'aux tribus. Seuls les États peuvent y prétendre. Cette situation est pour le moins paradoxale. Si l'État fédéral reconnaît l'autorité exclusive des institutions tribales sur les enfants amérindiens résidant dans une réserve, ces institutions ne peuvent toutefois accéder aux ressources fédérales qui permettraient d'exercer cette autorité, alors même que les enfants amérindiens, en tant que citoyens américains, ont bien droit à ces ressources. En effet, ils sont bien pris en compte par l'administration fédérale en charge des affaires sociales dans la répartition de l'argent fédéral entre les différents États fédérés, y compris les mineurs habitant dans les réserves.

Afin de dépasser les obstacles qui inhibent la circulation des aides sociales fédérales, l'Indian Child Welfare Act incite les États et les gouvernements tribaux à entrer dans un processus de négociation et à signer des accords intergouvernementaux grâce auxquels les premiers peuvent redistribuer l'argent fédéral aux seconds ${ }^{14}$. Au début du xxI ${ }^{\mathrm{e}}$ siècle, soixante et onze tribus et treize États ont ainsi négocié un accord relatif au titre IV-E du Social Security Act, lequel contribue notamment au remboursement des frais associés aux placements en familles d'accueil (Brown et al. 2000 : 75-76).

Ces accords intergouvernementaux ne sont pas sans poser problème. Tout d'abord, ils révèlent que les droits spécifiques reconnus par l'Indian Child Welfare Act ne sont pas garantis sur l'ensemble du territoire américain puisque l'application de la loi fédérale dépend du dialogue qui s'instaure entre les tribus et les États. Ensuite, ils se soldent souvent par une intégration des services sociaux tribaux aux dispositifs de protection de l'enfance des États. En d'autres termes, les normes qui régissent ces dispositifs fédérés s'appliquent désormais aux services sociaux tribaux, lesquels relèvent normalement du droit tribal. Or, ces normes ne sont pas nécessairement en adéquation avec les réalités sociétales au sein des tribus. Par exemple, les règles qui encadrent le recrutement des familles d'accueil au niveau des États sont souvent éloignées des dynamiques familiales amérindiennes et des conditions socio-économiques sur les réserves. Malgré la signature d'un accord intergouvernemental entre leur tribu et un État, de nombreuses familles d'accueil amérindiennes ne peuvent ainsi toujours pas accéder aux aides fédérales disponibles par le biais du titre IV-E du Social Security Act car elles ne rentrent pas dans les critères d'agrément fixés par l'État ${ }^{15}$.

\section{L'exception amérindienne sort des réserves}

Les problèmes d'accès aux ressources fédérales n'ont généralement pas permis aux tribus de mettre en place des services sociaux tribaux stables. En revanche, les dispositions prévues par la loi fédérale de 1978 ont eu pour conséquence de faire sortir l'exception amérindienne des réserves. En cela, la protection de l'enfance est un domaine remarquable. En effet, en établissant des compétences partagées entre les tribus et les États sur les procédures impliquant les enfants amérindiens qui résident en dehors des réserves, l'État fédéral reconnaît le lien essentiel qui unit ces enfants à la tribu à laquelle ils sont affiliés, où qu'ils résident dans l'espace américain. Si traditionnellement, les attributs de la spécificité autochtone sont conditionnés par la résidence dans la réserve, 
on peut dire que la protection de l'enfance contribue à désenclaver la question amérindienne, à étendre la sphère d'influence des institutions tribales et à rendre visible et significative cette spécificité sur l'ensemble du territoire étasunien.

La juge tribale des Ojibwe de la réserve de White Earth, dans le Minnesota, considère ainsi que les évolutions dans la sphère de la protection de l'enfance ont permis à de nombreux travailleurs sociaux et juges pour enfants au sein des États de se familiariser avec le statut amérindien et avec les réalités amérindiennes contemporaines qui, selon elle, sont encore souvent méconnus dans la société américaine, en particulier dans des régions à faible population amérindienne ${ }^{16}$. De même, au sein du comté de Hennepin (sur lequel s'étend la ville de Minneapolis) ${ }^{17}$, une équipe spécialisée dans les procédures impliquant des enfants amérindiens a été mise en place au milieu des années 1990. Pour mener à bien leur mission, les travailleurs sociaux qui composent cette équipe collaborent régulièrement avec les centres sociaux amérindiens de l'agglomération, mais également avec les institutions des tribus ojibwe et dakota avoisinantes, ainsi qu'avec celles des tribus plus lointaines auxquelles peuvent être affiliés les mineurs dont ils ont la responsabilité ${ }^{18}$. Ces employés du comté, qui pour la plupart ne sont pas amérindiens, coopèrent ainsi souvent avec des personnes issues de cultures qui n'ont pas nécessairement la même conception de la famille ou du bien-être de l'enfant. Au contact de ces personnes, leurs pratiques professionnelles évoluent. Cela se traduit par exemple par une prise en compte plus systématique des ressources existantes au sein des familles élargies qui, dans de nombreuses cultures amérindiennes, sont précisément les cellules destinées à procurer un sentiment de stabilité à l'enfant, au-delà des défaillances qui peuvent exister au niveau du couple parental. Les conceptions amérindiennes de la famille ou de l'enfance circulent ainsi de plus en plus parmi les professionnels américains de la protection de l'enfance.

\section{Crispations autour du statut amérindien}

Cependant, cette évolution génère beaucoup de crispations car ces conceptions sont parfois estimées incompatibles avec les normes de la société américaine. Afin d'illustrer notre propos, nous citerons l'exemple de jumelles nées en Californie qui, en 1993, sont placées en vue d'une adoption chez un couple résidant dans l'Ohio. Les parents biologiques, deux jeunes adultes non mariés, ont alors tous deux renoncé volontairement à leurs droits parentaux. Le père décide d'informer l'avocat chargé de l'adoption de ses origines amérindiennes. Toutefois, ce dernier explique que la procédure risque d'être plus longue et plus complexe si elle tombe sous le coup de l'Indian Child Welfare Act. Le père fait finalement le choix de ne pas révéler ses origines et la procédure suit son cours. Dans le même temps, la mère de cet homme, donc la grand-mère paternelle des jumelles, est mise au courant de la procédure d'adoption. Elle se tourne vers les autorités de la tribu Pomo, dont elle est membre, et effectue une demande d'affiliation tribale pour ses petites filles afin que la loi fédérale de 1978 puisse s'appliquer et que les enfants puissent être placés au sein de leur famille élargie. Les demandes d'affiliation sont acceptées et le gouvernement tribal dépose une requête auprès de la cour de première instance de Californie chargée de l'affaire, afin d'exercer son droit d'intervention. C'est à ce moment que le père décide de revenir sur son consentement initial à l'adoption. En 1995, le tribunal de Californie donne raison à la grand-mère et à la tribu. La procédure d'adoption est alors invalidée et les jumelles doivent être retirées de la garde des parents adoptifs. Ces derniers font cependant appel, et, un an plus tard, la cour d'appel du deuxième 
district de Californie renverse la décision du tribunal de première instance ${ }^{19}$. Finalement, les jumelles retournent auprès de leurs parents adoptifs.

21 Plusieurs litiges opposant ainsi la famille adoptive de l'enfant à un parent biologique amérindien ou à un membre de la famille élargie, soutenus par leur tribu, ont eu lieu ces dernières décennies. Le dernier en date est même parvenu jusqu'à la Cour suprême ${ }^{20}$. Ils témoignent tous de ce que Martine Ségalen (2010:12) a décrit par ailleurs comme un conflit entre « deux cultures de l'enfant » ou « deux façons de penser la place de l'enfant ${ }^{21}$ ». Pour les individus amérindiens et les tribus, l'enfant n'appartient pas seulement aux deux personnes qui composent le couple parental, qu'il s'agisse du couple de parents biologiques ou adoptifs, mais il est dès la naissance intégré dans un réseau de parenté plus large. Ainsi, le fait que des parents biologiques renoncent à leurs droits sur leur enfant n'implique pas que le lien d'appartenance soit rompu avec la famille élargie dans son ensemble. Faisant précisément référence au cas des jumelles, le juriste Marty Slaughter (2000 : 241) explique ainsi qu'au sein du droit tribal pomo, le père et ses filles "sont perçus comme des parties organiques de la famille tribale élargie». Pour les parents adoptifs, l'enfant appartient avant tout à un couple parental. Si le père et la mère choisissent de renoncer à leurs droits parentaux, les autres membres de la famille n'ont eux-mêmes plus de droits sur cet enfant. L'intervention de la grand-mère des jumelles est ainsi perçue comme empiétant sur les libertés fondamentales des individus au sein de la nation américaine.

22 Au terme de notre article, nous pouvons avancer que le statut particulier des enfants amérindiens aux États-Unis contribue à créer des situations de blocages dans la sphère de la protection de l'enfance. Le statut amérindien relève déjà en lui-même d'un processus d'exclusion puisque si d'un côté les cloisons entre les Amérindiens et les non-Indiens sont poreuses, c'est-à-dire que les Amérindiens ont aussi la nationalité américaine, de l'autre, en revanche, ces cloisons restent étanches. En effet, les non-Indiens ne peuvent prétendre au statut amérindien. Ces barrières juridiques sont la marque de l'existence de « nations dans la nation » américaine. De ce fait, l'adoption d'un enfant amérindien se rapproche, même si elle n'en est pas l'équivalent, d'une adoption internationale.

Les gouvernements tribaux sont devenus des acteurs essentiels dans l'organisation des services destinés aux mineurs amérindiens en danger. Il existe donc une répartition « tripartite » (Meyer 2002 : 39) des compétences et des responsabilités entre l'État fédéral, les États et les tribus. Or, nous l'avons vu, ces dernières ont du mal à accéder aux aides sociales fédérales, ce qui compromet leurs chances de fournir des services stables. Enfin, la reconnaissance, depuis l'Indian Child Welfare Act, du lien essentiel entre les enfants amérindiens et la tribu à laquelle ils sont affiliés a contribué à désenclaver la question amérindienne et à poser la question de la spécificité du statut amérindien en dehors des réserves. Ce processus s'accompagne d'une diffusion des conceptions amérindiennes de la famille ou de l'enfant parmi la société américaine mais, dans le même temps, génère des tensions. Celles-ci sont révélatrices de la difficile articulation entre droits constitutionnels (donc individuels) et droits spécifiques (donc collectifs) au sein de la démocratie étasunienne. 


\section{BIBLIOGRAPHIE}

Barsh, Russel, 1980, « The ICWA of 1878: A Critical Analysis », Hastings Law Journal, n 31, p. 1287-1336.

Bartholet, Elizabeth, 1995, « Race Separatism in the Family: More on Transracial Adoption Debate », Duke Journal of Gender Law \& Policy, vol. 2, n 1, p. 99-105.

Biolsi, Thomas, 2001, Deadliest Enemies: Law and the Making of Race Relations on and off Rosebud Reservation, Berkeley, University of California Press.

Brieland, D., 1973, « Far from Reservation: Transracial Adoption of American Indian Children », Social Service Review, vol. 47, $\mathrm{n}^{\circ}$ 2, p. 310-311.

Brown, Eddie, et al., 2000, Tribal/State Title IV-E Intergovernmental Agreements: Facilitating Tribal Access to Federal Resources, rapport de la Child Welfare Association.

Conseil tribal Navajo, 1960, Tribal Policy on Adoption of Navajo Orphans and Abandoned or Neglected Children, résolution du 18 novembre.

Corntassel, Jeff et Witmer, Richard, 2008, Forced Federalism: Contemporary Challenges to Indigenous Nationhood, Norman, the University of Oklahoma Press.

Deloria, Vine et Lytle, Clifford, [1978] 1998, The Nations Within: The Past and Future of American Indian Sovereignty, Austin, University of Texas Press.

Dorsey, Craig, 1993, « Adoption and the Indian Child Welfare Act », Youth Law News, vol. 14, p. 14-19.

Eachron, Ann Mac, Gustavsson, Nora, Cross, Suzanne et Lewis, Allison, 1996, « The Effectiveness of the Indian Child Welfare Act of 1978 ", Social Service Review, vol. 70, n 3, p. 451-463.

Fanshel, David, 1972, Far from the Reservation: The Transracial Adoption of American Indian Children, Metuchen, NJ, The Scarecrow Press.

Fletcher, Matthew, 2007, « Retiring the "Deadliest Enemies" Model of Tribal-State Relations ", Tulsa Law Review, $\mathrm{n}^{\circ}$ 47, p. 73-87.

Fletcher, Matthew, Singel, Wenona et Fort, Kathryn, 2009, Facing the Future: The Indian Child Welfare Act at 30, East Lansing, Michigan State University Press.

Fogg-Davies, Hawley, 2002, The Ethics of Transracial Adoption, Ithaca, Cornell University Press.

Gaber, Ivor (dir.), 1994, In the Best Interests of the Child: Culture, Identity and Transracial Adoption, Londres, Free Association Books.

Garner, Suzanne, 1993, «The Indian Child Welfare Act: A Review », Wicazo Sa Review, vol. 9, n 1, p. 47-51.

Hicks, Sarah, 2007, « Intergovernmental Relationships: Expressions of Sovereignty », dans M. Jorgensen (dir.), Rebuilding Native Nations: Strategies for Governance and Development, Tucson, University of Arizona Press, p. 251-268.

Johnson, Barbara, 1981, « The Indian Child Welfare Act of 1978: Implications for Practice », Child Welfare, vol. 60, $\mathrm{n}^{\circ} 7$, p. 435-446. 
Kennedy, Randall, 1994, "Orphans of Separatism: The Painful Politics of Transracial Adoption », American Prospects, $\mathrm{n}^{\circ}$ 17, p. 40-42.

Kessel, Jo Ann et Robbins, Susan, 1984, « The Indian Child Welfare Act: Dilemmas and Attitudes », Child Welfare, vol. 63, $\mathrm{n}^{\circ}$ 3, p. 225-232.

Lyslo, Arnold, 1968, The Indian Adoption Project - 1958 through 67, Report of Its Accomplishment, Evaluation and Recommendations for Adoption Services to Indian Children, Archives de la Child Welfare League of America, Boîte 17, Dossier, Indian Adoption Project 1963-1972, Social Welfare History Archives, University of Minnesota.

MacEachron, Ann, Gustavsson, Nora, Cross, Suzanne et Lewis, Allison, 1996, « The Effectiveness of the Indian Child Welfare Act of $1978 »$, Social Service Review, vol. 70, nº 3, p. 451-463.

Matheson, L., 1996, "The Politics of the Indian Child Welfare Act », Social Work, vol. 41, n² p. 232-235.

Maury, Françoise, 1999, L'Adoption interraciale, Paris, L'Harmattan.

Meyer, John (dir.), 2002, American Indians and U.S. Politics, A Companion Reader, Westport, Praeger.

Morgan Pryse, E., 1951, Lettre adressée au Children's Bureau et datée du 22 octobre, Records of the Children's Bureau, RG 102, Central File, Boîte 351, Dossier : Indians, National Archives at College Park.

Palmiste, Claire, 2011, L'Adoption d'enfants autochtones par des familles blanches aux USA : un cas de génocide culturel, Paris, Publibook.

Planchou, Céline, 2012, Entre exception et assimilation : le statut des Amérindiens aux États-Unis à travers le prisme de la protection de l'enfance, 1950-2008, Thèse de doctorat, Université Paris DiderotParis 7.

Ségalen, Martine, 2010, À qui appartiennent les enfants ?, Paris, Tallandier.

Slaughter, M. M., 2000, « Contested Identities: The Adoption of American Indian Children and the Liberal State ", Social and Legal Studies, vol. 9, $n^{\circ}$ 2, p. 227-248.

Subcommittee on Indian Affairs of the Committee on Interior and Insular Affairs, 1975, On Problems that American Indian Families face in Raising their Children and how these Problems are Affected by Federal Action or Inaction, U.S. Senate, $93^{\mathrm{e}}$ Cong., $2^{\mathrm{e}}$ Ses., 8-9 avril 1974, Wash. D.C., U.S. Government Printing Office.

Troy, Johnson, 1999, « The State and the American Indian: Who Gets the Indian Child? », Wicazo Sa Review, vol. 14, $\mathrm{n}^{\circ}$ 1, p. 197-214.

Unger, Steven (dir.), 1977, The Destruction of American Indian Families, New York, Association on American Indian Affairs.

Wilkins, David, 1994, « Reconsidering Tribal-State Compact Process », Policy Studies Journal, vol. $22, \mathrm{n}^{\circ} 3$, p. $474-488$.

\section{NOTES}

1. En particulier les titres IV-B «Child and Family Services» et IV-E «Federal Payments for Foster Care and Adoption Assistance ».

2. À sa création, en 1824, le Bureau des affaires indiennes était du ressort du ministère de la Guerre. C'est en 1849 qu'il a été transféré au ministère de l'Intérieur. 
3. Nous utilisons l'expression «adoption interraciale » en français (utilisée par Françoise Maury 1999) pour rendre compte de l'adoption dite transracial en anglais et communément désignée par l'acronyme TRA. Ces adoptions ont fait l'objet de nombreux ouvrages et articles aux États-Unis. Citons entre autres : Elizabeth Bartholet (1995), Hawley Fogg-Davies (2002), Ivor Gaber (1994) et Randall Kennedy (1994 : 40-42).

4. Peu de mécanismes existent alors qui permettent de placer un enfant, qu'ils soit amérindien ou non, dans un autre État. L'Indian Adoption Project a ainsi encouragé un processus de coordination inter-agence et d'harmonisation des pratiques de l'adoption sur l'ensemble du territoire américain. Deux ans après le lancement du projet apparaissent d'ailleurs les premières ébauches de l'Interstate Compact on the Placement of Children.

5. Dawes Act ou General Allotment Act, 24 Stat. 388, ch. 119, 25 USCA 331, 1887.

6. L'administration fédérale en charge des affaires indiennes usait de nombreux facteurs pour déterminer si un individu était « compétent » (competent) ou « incompétent » (incompetent). Nous citerons par exemple la manière avec laquelle un individu s'adaptait à la société américaine ou encore son " degré de sang indien » (Indian blood quantum). Précisons que la « compétence » d'un individu conditionnait à cette époque l'accession à la citoyenneté américaine.

7. L'ouvrage de Thomas Biolsi (2001) traite spécifiquement de ce processus sur la réserve lakota de Rosebud.

8. Citons par exemple le cas du Minnesota où les enfants amérindiens comptent pour $3,5 \%$ des placements en familles d'accueil et $12 \%$ des adoptions alors qu'ils représentent $0,8 \%$ de la population infantile de l'État. Dans le Dakota du Sud, les chiffres sont encore plus disproportionnés. Ainsi, $35 \%$ des adoptions et $56 \%$ des placements en famille d'accueil impliquent des mineurs amérindiens bien qu'ils comptent pour seulement un peu plus de $5 \%$ des enfants de la région. Les résultats de ces enquêtes ont été présentés devant le sous-comité aux Affaires indiennes du Sénat américain en 1974.

9. Le titre original est: «On Problems that American Indian Families Face in Raising their Children and how these Problems are Affected by Federal Action or Inaction ».

10. Voir par exemple les témoignages de William Byler, directeur de l'Association on American Indian Affairs (organisation non gouvernementale créée en 1946, lorsque plusieurs organisations non indiennes de soutien aux Amérindiens se regroupent, et qui défend les droits civiques, politiques et territoriaux des Indiens) ou du psychiatre Joseph Westermeyer.

11. Fisher v. District Court, 424 U.S. 382, 1976.

12. Indian Child Welfare Act, Public Law 95-608, 25 USC \$§1901-63, 1978. Cette loi fédérale a fait l'objet de nombreux articles, concernant notamment les problèmes qui entourent son application. Citons entre autres : Russel Barsh (1980), Suzanne Garner (1993), Barbara Johnson (1981), Jo Ann Kessel et Susan Robbins (1984), L. Matheson (1996), Ann Mac Eachron, Nora Gustavsson, Suzanne Cross et Allison Lewis (1996). Citons également l'ouvrage édité par Matthew Fletcher, Wenona Singel et Kathryn Fort (2009).

13. À l'exception de la santé qui, en 1954, a été transférée à l'Indian Health Service au sein du Department of Health and Human Services (alors appelé Department of Health, Education and Welfare). Une demande des Amérindiens au moment de l'adoption de l'Indian Child Welfare Act était d'ailleurs que ce soit l'agence fédérale en charge des affaires sociales, et non le BIA, qui soit chargé d'appliquer la loi, et notamment de distribuer les fonds spécifiquement débloqués par l'État fédéral.

14. Alors que les États ont été pendant très longtemps exclus de la relation spéciale entre les nations amérindiennes et l'État fédéral, ils sont désormais de plus en plus sollicités par ce dernier, lequel cherche ainsi à se décharger de certaines responsabilités (voir Jeff Corntassel et Richard Witmer 2008). Les relations entre les États et les gouvernements tribaux se sont ainsi multipliées durant les dernières décennies, indépendamment des évolutions dans la sphère de la protection de l'enfance. C'est toutefois le seul domaine, avec la réglementation des 
établissements de jeu, où ce dialogue est préconisé par une loi fédérale. La protection de l'enfance contribue ainsi à faire évoluer les modalités du dialogue entre les États et les tribus vers un modèle relationnel moins conflictuel. Sur les relations grandissantes entre ces deux entités politiques, voir les articles suivants: Matthew Fletcher (2007), Sarah Hicks (2007) et David Wilkins (1994).

15. C'est souvent le manque d'espace dans les habitations qui est mis en cause et qui bloque l'obtention de l'agrément.

16. Entretien de l'auteur avec Anita Fineday, White Earth Chief Judge, réserve ojibwe de White Earth, 5 décembre 2007. La juge Anita Fineday est membre de la tribu ojibwe de White Earth et exerce dans le Minnesota depuis 1989. Elle est diplômée de l'université du Colorado et de l'université d'Harvard. D'abord avocate, puis procureur pour la tribu de Leech Lake (1992-1995), elle devient juge principal de la cour tribale de White Earth en 1997.

17. Le dispositif de protection de l'enfance dans le Minnesota est décentralisé. Il repose ainsi avant tout sur les comtés.

18. Entretiens de l'auteur avec différents membres de l'équipe effectués à Minneapolis en octobre et novembre 2007. Cette même année, l'équipe spécialisée comprenait une quinzaine de personnes.

19. In re Bridget R., 41 Cal. App. 4th 1483, Californie, 1996.

20. Adoptive Couple v. Baby Girl, 570 U.S.__133 S. Ct. 2552, 2013.

21. Dans son ouvrage, Martine Ségalen oppose en fait la vision française (et plus généralement occidentale) à la vision tchadienne de l'enfant telles qu'elles se sont exprimées lors du cas de l'Arche de Zoé. Cette ONG française a voulu, en 2007, ramener en France des "orphelins » du Darfour et s'est vue accusée de vol par les autorités tchadiennes pour qui ces enfants n'étaient pas orphelins car, bien qu'ayant perdu leurs parents biologiques, ils n'en appartenaient pas moins à leur famille.

\section{RÉSUMÉS}

À la fois membres de la nation américaine et membres tribaux, les enfants amérindiens ont un statut particulier aux États-Unis. Ils relèvent ainsi en premier lieu de l'autorité de leur tribu. Au milieu du $\mathrm{xx}^{\mathrm{e}}$ siècle, les services sociaux des États interviennent de plus en plus au sein des familles amérindiennes et placent de nombreux enfants loin des réserves, au mépris de leur statut particulier. L'Indian Child Welfare Act (1978) change la donne et redonne tout son sens à ce statut dans la sphère de la protection de l'enfance. La loi fédérale contribue à faire sortir l'exception amérindienne des réserves mais révèle des crispations au sein de la nation américaine autour de la spécificité amérindienne.

Native American children enjoy a special status in the United States. They are both U.S. nationals and tribal members and as such, their well-being falls primarily under the authority of tribal institutions. In the middle of the twentieth century, tribal authority was often overridden by states and many Native children started to be placed in foster care or adoptive homes away from reservations, in violation of their special status. The Indian Child Welfare Act (1978) reaffirmed tribal authority over Native children wherever they reside on the U.S. territory. In doing so, it extended the expression of Native American special status beyond the limits of reservations but it also revealed tensions in the U.S. society over this status. 
INDEX

Mots-clés : Amérindiens, protection de l'enfance, adoption interraciale, enfants amérindiens, triangle État fédéral-États fédérés-tribus

Keywords : Native Americans, child welfare, transracial adoption, Native American children, The Federal-State-Tribal Triangle

\section{AUTEUR}

CÉLINE PLANCHOU

Université Paris 13, Sobonne Paris Cité, Pléiade (EA 7338) 